\title{
Clinical Profile of Non-neutropenic Patients with Invasive Candidiasis: A Retrospective Study in a Tertiary Care Center
}

\author{
Sowmya Sridharan ${ }^{1} \odot$, Ram Gopalakrishnan ${ }^{2} \odot$, Panchatcharam S Nambi $^{3}$, Suresh Kumar ${ }^{4}$, Nandini Sethuraman $^{5} \odot$, \\ V Ramasubramanian ${ }^{6}$
}

\begin{abstract}
Introduction: Invasive candidiasis (IC) is a major cause of morbidity and mortality in critically ill patients in the intensive care unit (ICU). In this study, we aim to analyze the clinical profile, species distribution, and susceptibility pattern of patients with IC.

Methods: Case records of non-neutropenic patients $\geq 18$ years of age with IC between January 2016 and June 2019 at a tertiary care referral hospital were analyzed. IC was defined as either candidemia or isolation of candida species from a sterile site (such as CSF; ascitic, pleural, or pericardial fluid; or pus or tissue from an intraoperative sample) in a patient with clinical signs and symptoms of infection.

Results: A total of 114 patients were analyzed, out of which 105 (92.1\%) patients had bloodstream infection (BSI) due to candida and 9 (7.9\%) had IC identified from a sterile site. Central line-associated blood stream infection (27 patients, 23.6\%) and a gastro-intestinal source (30 patients, $26.3 \%$ ) were the most common presumed sources for candidemia. The commonest species was Candida tropicalis 42 (36.8\%), followed by Candida glabrata 20 (17.5\%). Serum beta-D-glucan (BDG) was done only in 32 patients of the 114 (35.3\%); among those who were tested, 5 (15.6\%) had a BDG value of less than 80 pg/mL despite having Candida BSI. Fluconazole sensitivity was $69.5 \%$ overall. At 14 days after diagnosis of IC, $49.1 \%$ had recovered, with the remainder having an unfavorable outcome (32.4\% had died and $18.4 \%$ had left against medical advice).

Clinical significance: IC is a major concern in Indian ICUs, with a satisfactory outcome in only half of our patients. Serum BDG is a valuable test to diagnose blood culture-negative IC, but more studies are needed to determine its role in the exclusion of IC, as we had a small minority of patients with negative tests despite proven IC.

Conclusion: We recommend sending two sets of blood cultures and serum BDG assay for all suspected patients. Initiating empiric antifungal therapy with an echinocandin is advisable, in view of increasing azole resistance and the emergence of Candida auris, with de-escalation to fluconazole for sensitive isolates after clinical stability and blood culture clearance.

Keywords: Candidemia, Invasive candidiasis, Serum beta-D-glucan.

Indian Journal of Critical Care Medicine (2021): 10.5005/jp-journals-10071-23748
\end{abstract}

\section{INTRODUCTION}

Invasive candidiasis (IC) is a major cause of morbidity and mortality in critically ill patients in the intensive care units (ICUs) with an incidence of ICU-acquired candidemia of 6.8 cases per 1000 ICU admissions. ' Candidemia, defined as bloodstream infection (BSI) due to candida, can have a high mortality rate ranging from $38 \%$ to $75 \%$. ${ }^{1,2}$ Better identification techniques such as matrix-assisted laser desorption/ionization time-of-flight mass spectrometry (MALDI TOF-MS) for species identification and biomarkers such as serum beta-D-glucan (BDG) are now available in India. In the last decade, as there has been a rise in fluconazole resistance in candida species, and multidrug-resistant Candida auris has emerged, ${ }^{3,4}$ echinocandins are now generally considered the drugs of choice for IC. There are a few Indian studies that analyze the clinical features, details of the therapy, and outcome in the echinocandin/C. auris/MALDI-TOF/BDG era. We aimed to analyze the clinical profile of patients with IC in our center.

\section{Methods}

\section{Study Design}

A retrospective observational study of IC episodes between January 2016 and June 2019 was carried out at a 550-bed tertiary care referral hospital in Chennai, South India.

Definitions: IC was defined as an episode of candidemia with isolation of Candida spp. from at least one peripherally taken blood culture or from a sterile site (such as CSF; ascitic, pleural, or
${ }^{1-6}$ Department of Infectious Disease, Apollo Hospital, Chennai, Tamil Nadu, India

Corresponding Author: Sowmya Sridharan, Department of Infectious Disease, Apollo Hospital, Chennai, Tamil Nadu, India, Phone: +91 99662231818, e-mail: kesowmya@yahoo.com

How to cite this article: Sridharan S, Gopalakrishnan R, Nambi PS, Kumar S, Nandini S, Ramasubramanian V. Indian J Crit Care Med 2021;25(3):267-272.

Source of support: Nil

Conflict of interest: None

pericardial fluid; or pus or tissue from an intraoperative sample) in a non-neutropenic patient with clinical signs and symptoms of infection. Positive blood cultures from a patient were considered as being part of a single candidemia episode if they belonged to the same species and occurred less than 2 weeks apart. Any surgery done within 30 days preceding the onset of candidemia/IC was considered as a risk factor. Empiric therapy was defined as the administration of systemic antifungal drugs before blood culture reports are available. Definitive therapy was defined as the use of antifungal agents after the availability of blood culture reports.

\section{Inclusion Criteria}

Records of inpatients who were $\geq 18$ years of age with positive blood culture isolates for candida, and positive cultures from a sterile site 
(such as CSF; peritoneal, pleural, or pericardial fluid; or pus or tissue from an intraoperative sample) were included in the study.

\section{Exclusion Criteria}

Outpatients, patients $<18$ years, patients with absolute neutrophil count $<1000$ cells/ $\mu \mathrm{l}$, and those with cultures positive for candida from non-sterile sites such as endotracheal tube aspirates, urine, and sputum cultures were excluded.

\section{Data Collection and Interpretation of Results}

Study data were collected retrospectively based on medical records and chart review. Demographic variables, risk factors for IC (such as central line, ICU stay, receipt of broad-spectrum antibiotics, recent surgery), number of blood cultures sent, initiation of antifungal therapy, and clinical outcomes were recorded.

Blood culture was done using the BacT/ALERT system and species identification was performed by MALDI TOF (VITEK MS BioMérieux). These lab methods were standard lab procedures for the identification of the isolate. Antifungal susceptibility testing for fluconazole (FLU), voriconazole (VRC), amphotericin $B(A M B)$, caspofungin (CAS), micafungin (MFG), and flucytosine was performed using the AST-YSO8 card on the Vitek 2 system (BioMérieux). Results were interpreted as per the Clinical Laboratory Standards Institute guidelines. The US Food and Drug Administration-approved Fungitell ${ }^{\circledR}$ BDG assay was used to measure levels of the fungal biomarker serum 1,3-beta-D-glucan, with values $\geq 80 \mathrm{pg} / \mathrm{mL}$ being regarded as positive, and $<80 \mathrm{pg} / \mathrm{mL}$ as negative. Data entry was done in an MS Excel spreadsheet. All normally distributed continuous variables were expressed as mean \pm 2 SD. Non-normally distributed continuous variables were represented by median (interquartile range). Categorical variables were expressed as a percentage. Statistical analysis was done using IBM statistical package for the social sciences statistics 25.0.

The study was cleared by the Hospital Ethical Committee before commencement.

\section{Results}

A total of 114 patients were analyzed, out of whom 105 (92.1\%) patients had BSI due to candida (Table 1) while 9 (7.9\%) had IC identified from a sterile site (Table 1). Fifty-eight (50.9\%) patients were hospitalized elsewhere before being transferred to our center. And 102 patients (89.5\%) required ICU stay with a median duration of 13.5 days (interquartile range [IQR], 5 to 25.5) (Table 2). Comorbidities are shown in Table 2. Seventy-five (65.8\%) patients had acute kidney injury and around the same number, 76 (66.7\%) had a septic shock with 52 (45.6\%) having a SOFA score more than or equal to 7 (Table 2). Seventy-six (66.7\%) patients had central venous catheters (CVCs) at the time of IC suspicion, out of which 48 (42.1\%) had catheter removal. Central line-associated bloodstream infection, CLABSI (27 patients, 23.6\%), presumed GI source (30 patients, $26.3 \%$ ), and a presumed urinary source (11 patients, $9.6 \%$ ) were the most commonly suspected sources for the IC event.

Figure 1 shows the species distribution of our isolates. Out of 114 patients with IC, blood cultures were done for 110 (96.5\%) patients, out of whom 2 sets of blood cultures were done for 95 $(86.3 \%)$ patients. The average number of days after admission to culture positivity in patients with IC was 6.6 days. The yield was maximum in the aerobic bottle $(106,96.7 \%)$ whereas $4(3.6 \%)$ flagged only in the anaerobic bottle. The average time to culture positivity for patients with candida BSI was 79 hours (Table 3).
Table 1: Demographic distribution of patients with IC

\begin{tabular}{|c|c|}
\hline $\begin{array}{l}\text { Total number of patients included in } \\
\text { the study }\end{array}$ & 114 \\
\hline $\begin{array}{l}\text { Number of patients with IC due to } \\
\text { Candida BSI }\end{array}$ & $105(92.1 \%)$ \\
\hline $\begin{array}{l}\text { Number of patients with IC due to } \\
\text { SSC }\end{array}$ & $9(7.9 \%)$ \\
\hline Median age in years (IQR) & $58(49-68)$ \\
\hline Sex & $\begin{array}{l}\text { - Female: } 38(33.3 \%) \\
\text { - Male: } 76(66.7 \%)\end{array}$ \\
\hline Transferred from another hospital & $\begin{array}{l}\text { - } \text { Yes: } 58(50.9 \%) \\
\text { - } \text { No: } 47(41.2 \%) \\
\text { - } \text { Details unknown: } 9(7.9 \%)\end{array}$ \\
\hline ICU stay & $\begin{array}{l}\text { - Yes: } 102(89.5 \%) \\
\text { - No: } 12(10.5 \%)\end{array}$ \\
\hline $\begin{array}{l}\text { Total duration of hospital stay in days } \\
\text { (IQR) }\end{array}$ & $11.5(4.75-22)$ \\
\hline Duration of ICU stay in days (IQR) & $13.5(5-25.25)$ \\
\hline \multicolumn{2}{|l|}{ Comorbidities } \\
\hline DM & 60 (52.6\%) \\
\hline HT & $53(46.5 \%)$ \\
\hline Solid tumours & $6(5.3 \%)$ \\
\hline Chronic kidney disease & $19(16.7 \%)$ \\
\hline Chronic liver disease & $8(7 \%)$ \\
\hline \multicolumn{2}{|l|}{ Source of IC } \\
\hline CLABSI & 27 (23.7\%) \\
\hline $\begin{array}{l}\text { Presumed intraabdominal source } \\
\text { (Gl surgery, pancreatitis, etc.) }\end{array}$ & $30(26.3 \%)$ \\
\hline Urine & 11 (9.6\%) \\
\hline Infective endocarditis & $1(0.9 \%)$ \\
\hline Postneurosurgical intervention & $3(2.6 \%)$ \\
\hline Source unclear & $42(36.8 \%)$ \\
\hline
\end{tabular}

Repeat blood cultures to look for clearance were done in 68 patients (64.7\%). For 29 patients (27.6\%), repeat cultures could not be obtained as patients either died or asked for discharge against medical advice (DAMA). Of those for whom repeat cultures were done, it was observed that $46(67.6 \%)$ had cleared on the first instance (within 72 hours), while 22 (32.3\%) required 2 or more subsequent cultures for clearance.

Serum BDG was done only in 32 of the 114 patients (35.3\%); among those who were tested, 21 had a positive value (Fig. 2). Five of 32 patients (15.6\%) had a negative BDG value of less than $80 \mathrm{pg} /$ $\mathrm{mL}$ despite having IC. Among those five with negative BDG, Candida tropicalis was isolated in four and Candida glabrata in one. In the same group, one patient died, one was discharged against advice, and three were discharged after improvement.

Screening echocardiogram and dilated fundus examination for candida BSI patients were done for 61 (58\%) and 28 (26.6\%), with infective endocarditis being identified in two patients (Table 4). For 29 (27.6\%), screening echocardiogram and fundus examination could not be done as they died or left against advice within 48 hours. The antifungal susceptibilities of candida BSI are listed in Table 5A. Fluconazole sensitivity was $69.5 \%$ overall while echinocandins sensitivity was $100 \%$. All isolates of Candida albicans and $94.8 \%$ of $C$. tropicalis were azole sensitive (Table 5B).

Among those with candida BSI, 39 patients were started on fluconazole, 54 on echinocandin (43 on caspofungin), and 2 on amphotericin B. On analyzing the outcome at the end of 14 days, 
Table 2: Risk factor distribution for IC

\begin{tabular}{|c|c|}
\hline Variable & Number of patients (percentage) \\
\hline Immunosuppressive therapy & - $14(12.3 \%)$ \\
\hline Solid-organ transplant & $\begin{array}{l}\text { - Renal transplant: } 6(5.3 \%) \\
\text { - Heart transplant: } 2(1.8 \%) \\
\text { - } \text { Liver transplant: } 1(0.9 \%) \\
\text { - Heart and lung transplant: } \\
1(0.9 \%) \\
\text { - No transplant: } 104(91.2 \%)\end{array}$ \\
\hline Acute kidney injury (AKI) & $\begin{array}{l}\text { - No AKI: } 39(34.2 \%) \\
\text { - AKI present: } 75(65.8 \%) \\
\text { - AKI patients requiring dialy- } \\
\text { sis: } 14 \text { patients }\end{array}$ \\
\hline SOFA score & $\begin{array}{l}\cdot \quad<7: 37(32.4) \\
\cdot \quad \geq 7: 52(45.6 \%) \\
\text { - Not available: } 25 \text { (21.9) }\end{array}$ \\
\hline Central venous catheter & $\begin{array}{l}\text { - No line: } 38(33.3 \%) \\
\text { - Line present: } 76(66.7 \%) \\
\text { - C line removed after CLABSI/ } \\
\text { IC suspicion: } 48(42.1 \%)\end{array}$ \\
\hline Foley catheter & $73(64 \%)$ \\
\hline Total parenteral nutrition (TPN) & $\begin{array}{l}\text { - NO TPN: } 104 \text { (91.2\%) } \\
\text { - TPN: } 8(7 \%) \\
\text { - Feeding jejunostomy (FJ) } \\
\text { FEEDS: } 1(0.9 \%) \\
\text { - Percutaneous endoscopic } \\
\text { gastrostomy (PEG): } 1 \text { (0.9\%) }\end{array}$ \\
\hline Surgery & $\begin{array}{l}\text { - No surgery: } 69(60.5) \\
\text { - Non-Gl surgery: } 28(24.6 \%) \\
\text { - Gl surgery: } 17(14.9)\end{array}$ \\
\hline $\begin{array}{l}\text { Prior antibiotics usage (intravenous } \\
\text { antibiotic used for at least } 3 \text { days } \\
\text { within the previous } 1 \text { week) }\end{array}$ & - $114(100 \%)$ \\
\hline Bacteremia & $\begin{array}{l}\text { - Concomitant bacteremia: } \\
5(4.4 \%) \\
\text { - Previous bacteremia: } \\
25(21.9 \%) \\
\text { - No bacteremia: } 84(73.7 \%)\end{array}$ \\
\hline $\begin{array}{l}\text { Prior antifungal exposure for at } \\
\text { least }>3 \text { days (in last } 14 \text { days) }\end{array}$ & $\begin{array}{l}\text { - No exposure: } 91(79.8 \%) \\
\text { - Fluconazole: } 21(18.4 \%) \\
\text { - Amphotericin B: } 1(0.9 \%) \\
\text { - Echinocandin: } 1(0.9 \%)\end{array}$ \\
\hline
\end{tabular}

it was observed that 37 (32.4\%) had died, 21 (18.4\%) had requested DAMA, and 56 (49.1\%) had improved or been discharged with appropriate outpatient therapy (Fig. 3).

\section{Discussion}

Candida is a part of the normal microbial flora of the skin and the gut but can become an invasive pathogen in the presence of various risk factors. In a retrospective study done in Italy, it was noted that recent ICU stay, the CVC, parenteral nutrition, major abdominal surgery, diabetes, and immunosuppression were all major risk factors for IC. ${ }^{5}$ In our study, two-thirds of the patients had a CVC, with 26 (22.8\%) with IC secondary to CLABSI. The traditional risk factors for IC such as CVC, broad-spectrum antibiotics, and abdominal surgeries were all found in our study also. Candidemia was diagnosed in an average of 6.6 days after admission, more than a day earlier than noted in an Indian multi-center study. ${ }^{1}$ On comparing the average days of onset of candidemia from the day of

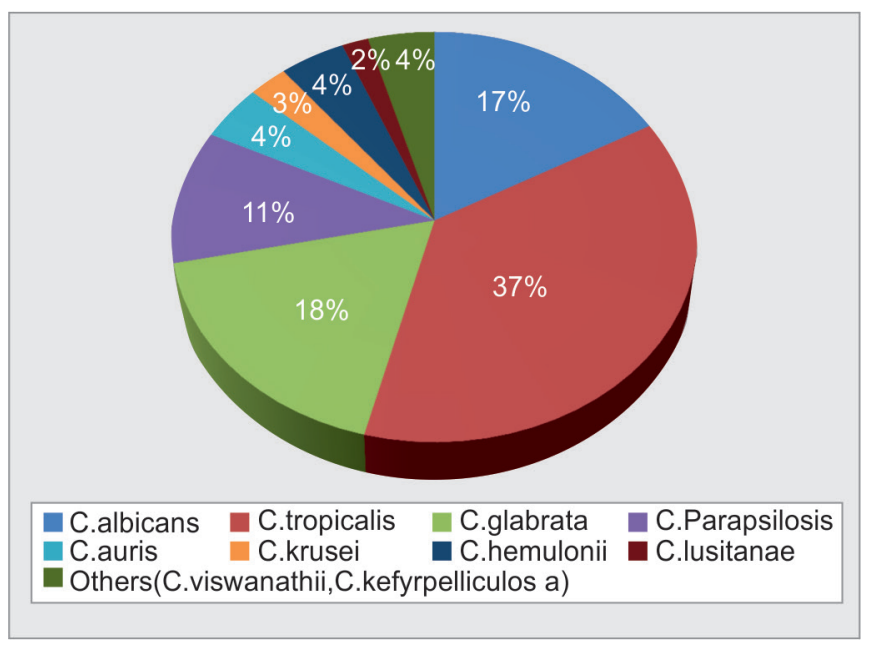

Fig. 1: Species distribution of patients with IC

Table 3: Blood culture analysis of patients with candida BSI

\begin{tabular}{|c|c|}
\hline $\begin{array}{l}\text { Average number of days after } \\
\text { admission to culture positivity in } \\
\text { patients with IC }\end{array}$ & 6.6 days \\
\hline $\begin{array}{l}\text { Average time to positivity of blood } \\
\text { culture after blood draw }\end{array}$ & $79 \mathrm{hrs}$ \\
\hline $\begin{array}{l}\text { Blood cultures on suspicion of } \\
\text { sepsis/IC }\end{array}$ & $\begin{array}{l}\text { - Sent: } 110(96.5 \%) \\
\text { - Not sent: } 4(3.5 \%)\end{array}$ \\
\hline $\begin{array}{l}\text { Number of sets of blood cultures } \\
\text { done }\end{array}$ & $\begin{array}{l}\text { - } 1 \text { set }(2 \text { bottles): } 11(11 \%) \\
\text { - } 2 \text { sets ( } 4 \text { bottles): } 95(86.3 \%) \\
\text { - } 3 \text { sets ( } 6 \text { bottles): } 3(2.7 \%)\end{array}$ \\
\hline Number of aerobic bottles positive & $\begin{array}{l}\text { - } 2 \text { bottles: } 48(43.6 \%) \\
\text { - } 3 \text { bottles: } 3(2.7 \%) \\
\text { - } 1 \text { bottle: } 55(50.0 \%) \\
\text { - } 0 \text { bottle: } 4(3.6 \%)\end{array}$ \\
\hline $\begin{array}{l}\text { Number of anaerobic bottles } \\
\text { positive }\end{array}$ & $\begin{array}{l}\text { - } 0 \text { bottle: } 99(90 \%) \\
\text { - } 1 \text { bottle: } 9(8.1 \%) \\
\text { - } 2 \text { bottles: } 2(1.8 \%) \\
\text { - } 3 \text { bottles: } 0(0 \%)\end{array}$ \\
\hline
\end{tabular}

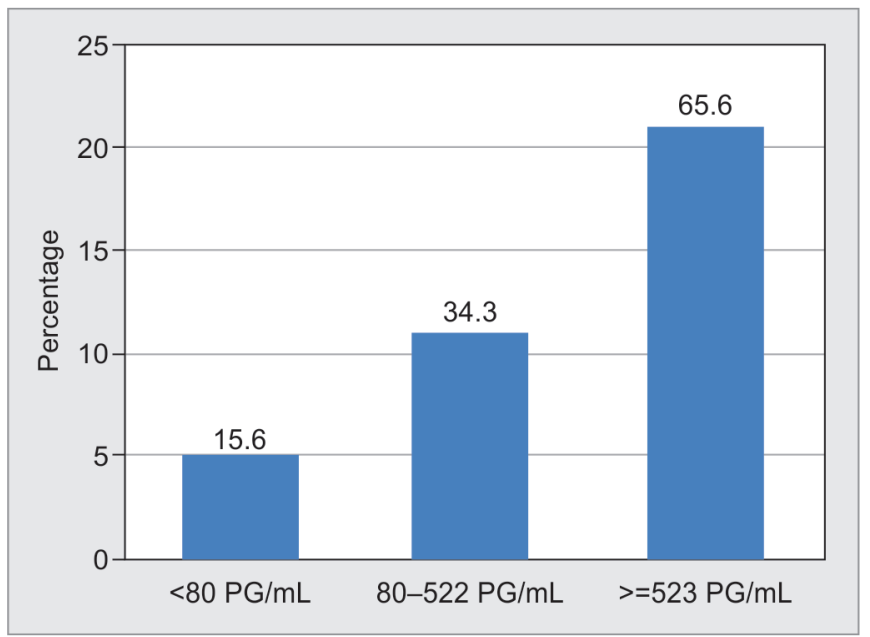

Fig. 2: Values of serum BDG in 32 patients with IC 
Table 4: Clinical and treatment characteristics of patients with IC

\begin{tabular}{ll}
\hline Empiric antifungal started & - Fluconazole: $46(40.4 \%)$ \\
when IC suspected & - Echinocandins: $66(59.9 \%)$ \\
Initiation of antifungal & - Amphotericin B: $2(1.8 \%)$ \\
therapy & - Eulture based: $19(16.7 \%)$ \\
& Empiric: $54(47.4 \%)$ \\
& $41(36 \%)$ \\
Switch to fluconazole in & - De-escalated: $19(28.7 \%)$ \\
patients on empiric & - No change: $47(71.2 \%)$
\end{tabular}
echinocandin after DST availability

Repeat blood cultures for clearance in patients with candida BSI

- Not done: 5 (4.7\%)

- Done: 68 (64.7\%)

- Not done due to death/discharge against advice: 34 (32.3\%)

Clearance achieved with repeat blood culture

- Cleared: $46(67.6 \%)$

- Not cleared: 22 (32.3\%)

Echocardiogram for patients - Done and reported normal: with Candida BSI 59 (56.1\%)

- Done with Infective endocarditis identified: 2 (1.9\%)

- Not done (reason not identified): 20 (19\%)

- Not done due to death: $16(15.2 \%)$

- Not done due to DAMA: 8 (7.6\%)

Dilated fundus examination - Done and reported normal: 28 (26.6\%)

- Not done (reason not identified): $43(40.9 \%)$

- Not done due to death: 19 (18.0\%)

- Not done due to DAMA: 15 (14.2\%)

admission, there was no difference among those who had a history of transfer from another hospital and those who did not.

The aerobic bottle of the blood culture sets flagged almost always, suggesting that two aerobic bottles could potentially replace the traditional aerobic/anaerobic set. The average time to flag was longer than 3 days, emphasizing the need for additional tests such as biomarkers and empiric therapy pending culture reports. As with other Indian studies and similar to our previous study, it was observed that $C$. tropicalis was the commonest species causing IC in $36.8 \%$ of the patients. ${ }^{6-8}$ However, in comparison to our earlier study, fluconazole susceptibility fell from $77.5 \%$ to $69.5 \%{ }^{6}$ and prior antifungal exposure increased from $15.2 \%$ to $19.2 \%$. All our isolates were susceptible to echinocandins. C. auris, an emerging opportunistic pathogen, presents major challenges in hospitalized patients in terms of identification, multidrug resistance, and infection control. VITEK 2 misidentifies $C$. auris as Candida haemulonii, Candida famata, Saccharomyces cerevisiae, or Rhodotorula glutinis. Only $4.7 \%$ of our isolates were C. auris.

Our study builds on our experience with serum BDG in patients with candidemia. ${ }^{9}$ Serum BDG was elevated in the vast majority of our patients with IC, though they were done only in about a third of the study subjects as serum BDG was introduced in the year 2017 in our center. This supports its use as a valuable adjunct in the diagnosis of IC when blood cultures are negative. Five (15.6\%) of 32 patients had a negative value despite proven IC, mostly with $C$. tropicalis. This is contrary to two previously published studies, which concluded that
Table 5A: Antifungal susceptibility for candida BSI

\begin{tabular}{lcl}
\hline Antifungal & Number of susceptible isolates & $\begin{array}{l}\text { Number of isolates } \\
\text { intermediate }\end{array}$ \\
\hline Fluconazole & $73(69.5 \%)$ & $9(8.5 \%)$ \\
Echinocandin & $105(100 \%)$ & 0 \\
Voriconazole & $97(92.3 \%)$ & $3(2 \%)$ \\
Amphotericin B & $85(80.9 \%)$ & $6(5.7 \%)$ \\
\hline
\end{tabular}

Table 5B: Species wise distribution of fluconazole susceptibility

\begin{tabular}{llll}
\hline & $\begin{array}{l}\text { Number of } \\
\text { susceptible isolates } \\
\text { (\% within species) }\end{array}$ & $\begin{array}{l}\text { Number } \\
\text { of isolates } \\
\text { resistant }\end{array}$ & $\begin{array}{l}\text { Number of } \\
\text { isolates } \\
\text { intermediate }\end{array}$ \\
\hline C. tropicalis & $37(94.8 \%)$ & $1(2.5 \%)$ & $1(2.5 \%)$ \\
C. glabrata & $5(26.3 \%)$ & $9(47.4 \%)$ & $5(26.3 \%)$ \\
C. albicans & $17(100 \%)$ & 0 & 0 \\
C. parapsilosis & $7(58.3 \%)$ & $4(33.3 \%)$ & $1(8.3 \%)$ \\
C. auris & 0 & $4(100 \%)$ & 0 \\
C. hemulonii & 0 & $3(60 \%)$ & $2(40 \%)$ \\
C. krusei & $1(33.3 \%)$ & $2(66.7 \%)$ & 0 \\
C. lusitanae & $2(100 \%)$ & 0 & 0 \\
Others & $4(100 \%)$ & 0 & 0 \\
\hline
\end{tabular}

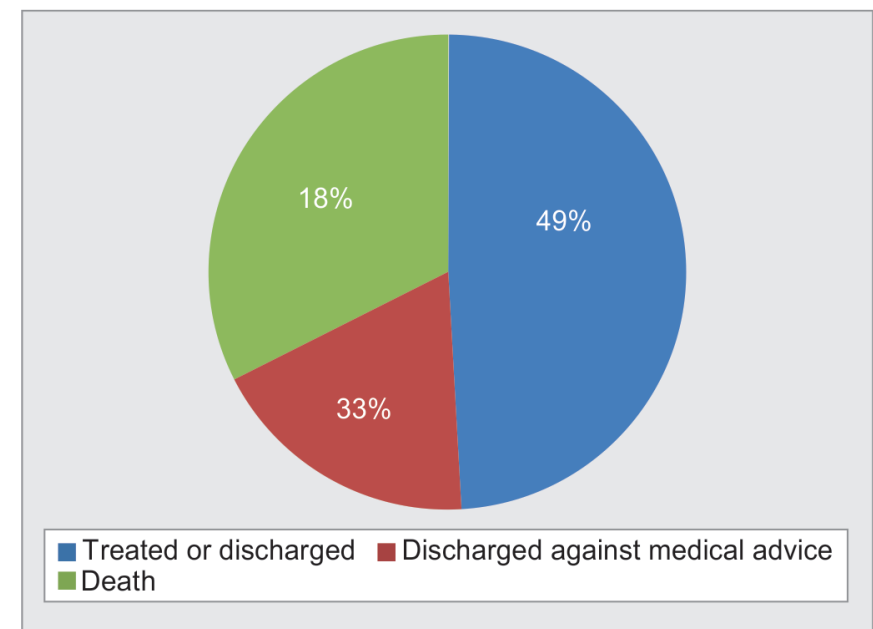

Fig. 3: Outcome at the end of 14 days for patients with IC

IC can be excluded if serum BDG values are less than $80 \mathrm{pg} / \mathrm{mL}$., 10 Agnelli et al. concluded that BDG-negative patients with IC may have a better prognosis due to a lower fungal burden. ${ }^{11}$ Our findings suggest that a negative BDG does not exclude IC in a patient with a high pretest probability for IC who responds to antifungal agents and this be factored into clinical decision making (Fig. 4).

The majority of our patients were treated in concordance with standard guidelines that recommend looking for blood culture clearance, echocardiogram, and dilated fundus examination. ${ }^{12,13}$ In our study, the majority of those with candida BSI had a repeat culture done of which had cleared. Only two were reported to have infective endocarditis and none had ocular candidiasis, significantly less than previous studies. ${ }^{14}$ 


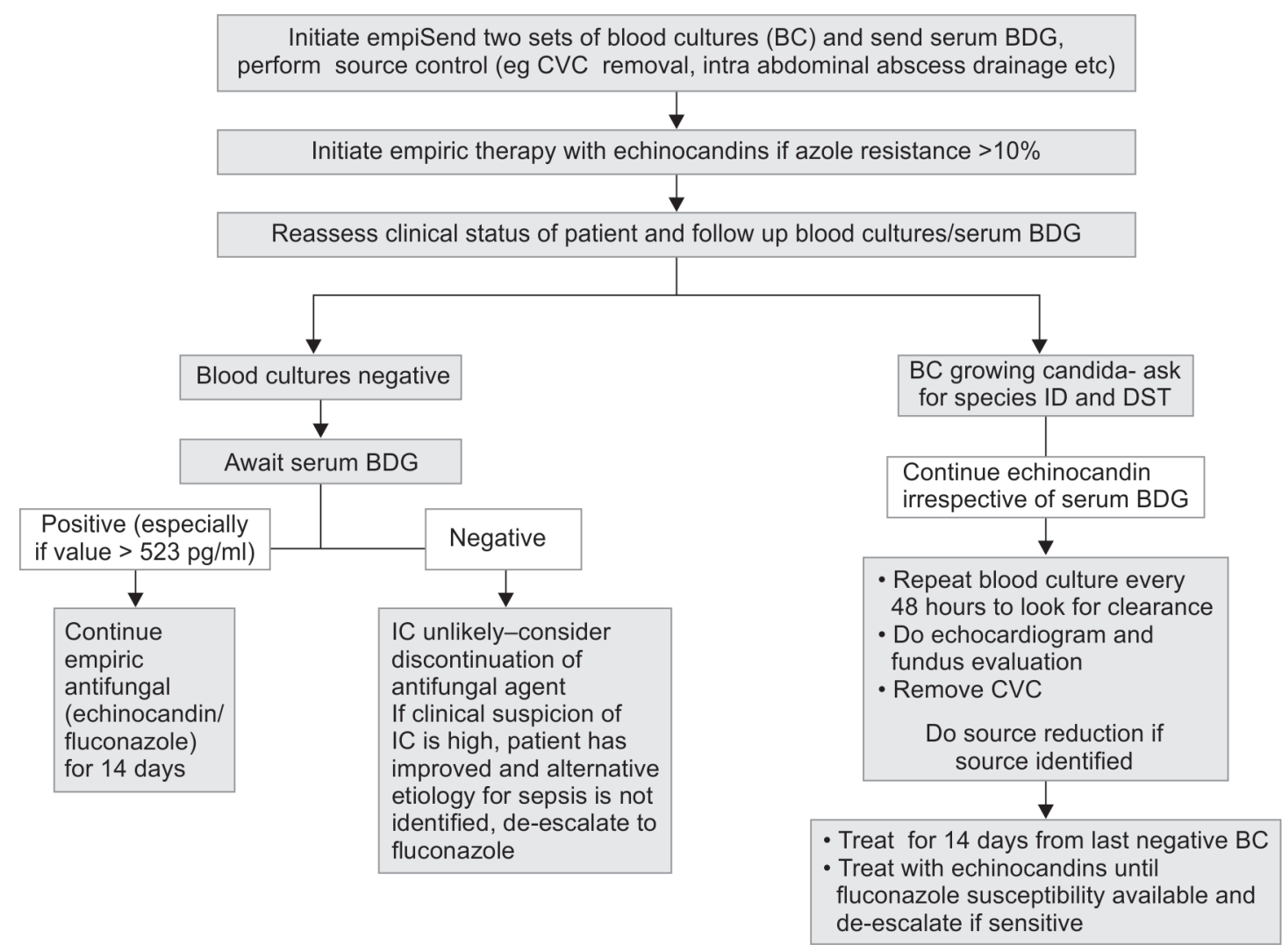

Fig. 4: Algorithm for non-neutropenic patient with suspected IC in the ICU

More patients received echinocandins than fluconazole for therapy in our study. The overall fall in fluconazole susceptibilities and rising prevalence of $C$. auris favor starting therapy with echinocandins, as recommended by guidelines. ${ }^{12}$ Crude mortality at the end of 14 days was around $32.4 \%$ in our study, which was lower than in studies from other tertiary care centers worldwide. ${ }^{15}$ However, an additional $18.4 \%$ of the patients left the hospital against medical advice due to the critical nature of the patient's illness, financial constraints, or other reasons. Assuming a worstcase scenario for the patients to be death at the end of 14 days, our rate of poor outcome is $50.8 \%$, emphasizing the need for early diagnosis and appropriate therapy. ${ }^{16}$

Our study has several limitations. First, this was a retrospective single-center experience. Second, serum BDG was done only for a minority as per the treating clinician's discretion. Third, we did not have to follow up data for almost one-fifth of the patients as they left against advice.

\section{Conclusion}

IC is a major concern in Indian ICUs, with a satisfactory outcome in only half of our patients. Our study reiterates known facts about IC, such as risk factors, shift to a non-albicans spectrum, and increasing azole resistance. Serum BDG is a valuable test to diagnose blood culturenegative IC, but more studies are needed to determine its role in the exclusion of IC, as we had a small minority of patients with negative tests despite proven IC. We recommend sending two sets of blood cultures and serum BDG assay for all suspected patients and initiating empiric antifungal therapy. Echinocandins are preferred choices for initial empiric therapy in view of increasing azole resistance and the emergence of $C$. auris, with de-escalation to fluconazole for azole sensitive isolates after clinical stability and clearance of blood cultures.

\section{OrCID}

Sowmya Sridharan 가 http://orcid.org/0000-0002-8097-3261

Ram Gopalakrishnan @ http://orcid.org/0000-0002-2263-1861

S Nandini @i http://orcid.org/0000-0002-3803-5511

\section{References}

1. Chakrabarti A, Sood P, Rudramurthy SM, Chen S, Kaur H, Capoor M, et al. Incidence, characteristics and outcome of ICU-acquired candidemia in India. Intensive Care Med 2015;41(2):285-295. DOI: 10.1007/s00134-014-3603-2.

2. Gupta P, Prateek S, Chatterjee B, Kotwal A, Singh AK, Mittal G. Prevalence of Candidemia in ICU in a tertiary care hospital in North India. Int J Curr Microbiol Appl Sci 2015;4(6): 566-575.

3. Jeffery-Smith A, Taori SK, Schelenz S, Jeffery K, Johnson EM, Borman A, et al. Candida auris: a review of the literature. Clin Microbiol Rev 2017;31(1):e00029-17. DOI: 10.1128/CMR.00029-17.

4. Oberoi JK, Wattal C, Goel N, Raveendran R, Datta S, Prasad K. Nonalbicans Candida species in blood stream infections in a tertiary care hospital at New Delhi, India. Indian J Med Res 2012;136(6):997-1003.

5. Bassetti M, Merelli M, Ansaldi F, de Florentiis D, Sartor A, Scarparo C, et al. Clinical and therapeutic aspects of candidemia: a five year single centre study. PLoS One 2015;10(5):e0127534. DOI: 10.1371/journal. pone. 0127534 .

6. Rajalakshmi A, Shareek PS, Sureshkumar D, Gopalakrishnan R, Yamuna E, Ramasubramanian V. Candidemia species distribution and emergence of Candida haemulonii complex isolates resistant tofluconazole in South India. J Contemp Clin Pract 2018;4(2):47-52. DOI: $10.18683 /$ jccp.2018.1035. 
7. Bhattacharjee P. Epidemiology and antifungal susceptibility of Candida species in a tertiary care hospital, Kolkata, India. Curr Med Mycol. 2016;2(2):20-27. DOI: 10.18869/acadpub.cmm.2.2.5.

8. Tak V, Mathur P, Varghese P, Gunjiyal J, Xess I, Misra MC. The epidemiological profile of candidemia at an Indian trauma care center. J Lab Physicians 2014;6:96-101. DOI: 10.4103/0974-2727.141506.

9. Bansal N, Gopalakrishnan R, Sethuraman N, Ramakrishnan N, Nambi PS, Kumar DS, et al. Experience with $\beta$-D-glucan assay in the management of critically ill patients with high risk of invasive candidiasis: an observational study. Indian J Crit Care Med 2018;22(5):364-368. DOI: 10.4103/ijccm.IJCCM_4_18.

10. Del Bono V, Delfino E, Furfaro E, Mikulska M, Nicco E, Bruzzi P, et al. Clinical performance of the $(1,3)-\beta$-D-glucan assay in early diagnosis of nosocomial Candida bloodstream infections. Clin Vaccine Immunol 2011;18(12):2113-2117. DOI: 10.1128/CVI.05408-11.

11. Agnelli C, Bouza E, del Carmen Martínez-Jiménez M, Navarro R, Valerio $M$, Machado $M$, et al. Clinical relevance and prognostic value of persistently negative (1,3)- $\beta$-D-glucan in adults with candidemia: a 5-year experience in a tertiary hospital. Clin Inf Dis 70(9):1925-1932. DOI: 10.1093/cid/ciz555.

12. Pappas PG, Kauffman CA, Andes DR, Clancy CJ, Marr KA, OstroskyZeichner $L$, et al. Clinical practice guideline for the management of candidiasis: 2016 update by the Infectious Diseases Society of America. Clin Inf Dis 2016;62(4):e1-e50. DOI: 10.1093/cid/ civ933.

13. Martin-Loeches I, Antonelli M, Cuenca-Estrella M, Dimopoulos G, Einav S, De Waele, JJ, et al. ESICM/ESCMID task force on practical management of invasive candidiasis in critically ill patients. Intensive Care Med 2019;45:789-805. DOI: 10.1007/s00134-01905599-w.

14. Rodríguez-Adrián LJ, King RT, Tamayo-Derat LG, Miller JW, Garcia $\mathrm{CA}, \mathrm{Rex} \mathrm{JH}$. Retinal lesions as clues to disseminated bacterial and candidal infections: frequency, natural history, and etiology. Med (Baltim) 2003;82:187-202. DOI: 10.1097/01.md.0000076008. 64510.f1.

15. Xiao Z, Wang Q, Zhu F, An Y. Epidemiology, species distribution, antifungal susceptibility and mortality risk factors of candidemia among critically ill patients: a retrospective study from 2011 to 2017 in a teaching hospital in China. Antimicrob Resist Infect Control 2019;8:89. DOI: 10.1186/s13756-019-0534-2.

16. Koehler P, Stecher M, Cornely OA, Koehler D, Vehreschild MJGT, Bohlius J, et al. Morbidity and mortality of candidae-mia in Europe: an epidemiologic meta-analysis. Clin Microbiol Infect 2019;25:12001212. DOI: 10.1016/j.cmi.2019.04.024. 